PROCEEDINGS OF THE

AMERICAN MATHEMATICAL SOCIETY

Volume 138, Number 11, November 2010, Pages 3945-3955

S 0002-9939(10)10380-3

Article electronically published on May 7, 2010

\title{
UNIVERSALITY AND ULTRADIFFERENTIABLE FUNCTIONS: FEKETE'S THEOREM
}

\author{
A. MOUZE AND V. NESTORIDIS \\ (Communicated by Nigel J. Kalton)
}

\begin{abstract}
The purpose of this article is to establish extensions of Fekete's Theorem concerning the existence of universal power series of $C^{\infty}$ functions defined by estimates on successive derivatives.
\end{abstract}

\section{INTRODUCTION}

Before 1914 Fekete showed that there exists a formal real power series $\sum_{n=1}^{+\infty} a_{n} x^{n}$ such that for every continuous function $h$ on $[-1,1]$ with $h(0)=0$, there exists an increasing sequence $\left(\lambda_{n}\right)$ of positive integers such that

$$
\sup _{x \in[-1,1]}\left|\sum_{k=1}^{\lambda_{n}} a_{k} x^{k}-h(x)\right| \rightarrow 0 \text { as } n \rightarrow+\infty
$$

(see [13). This result is, as far as we know, the first result about universal Taylor series. Since then, many results on universal series have appeared (see for instance [15, 4], 10] or 11]). The first proofs of the existence of universal series were constructive. In [6] it was proven that all the previous universalities were generic. Thus the use of Baire's Theorem yields a first simplification of the proofs. Furthermore it appears that the proofs need to exhibit a polynomial which approximates both a given function in the space where the universal function should live and a given function in the space where the universal property holds. Combining this fact with Baire's Theorem, Bayart, Grosse-Erdmann, Nestoridis and Papadimitropoulos in 1] develop an abstract theory of universal series, from which they deduce easily and in a unified way the existing results as well as new statements. In particular they obtain results about universal expansions of $C^{\infty}$ functions on arbitrary open subsets of $\mathbb{R}^{l}(l \geq 1)$, as well as an extension of Fekete's Theorem at the level of formal power series in $\mathbb{R}^{l}$.

Our aim is to obtain universal expansions of ultradifferentiable functions. Let us recall that ultradifferentiable functions are $C^{\infty}$ functions with a control on the growth of all derivatives (see Definitions 2.8 and 3.3). Such functions appeared as natural solutions of certain partial differential equations. In this paper we deal with non-quasianalytic classes (such as Gevrey classes), where the Borel theorem holds

Received by the editors June 25, 2009 and, in revised form, December 27, 2009 and January 12 , 2010

2010 Mathematics Subject Classification. Primary 30K05, 41A58, 26E10.

Key words and phrases. Universal series, Taylor series, infinitely differentiable real functions.

(C) 2010 American Mathematical Society 
14] (see [3] and [17 too). First we apply the abstract theory of universal series in a space of sequences of Beurling type, which is a Baire space. The combination of this new result with Borel's Theorem in the space of ultradifferentiable functions ensures the existence of universal elements in the Baire space of $C^{\infty}$ functions of Beurling type. These results allow us to exhibit universal elements in another space of ultradifferentiable functions (functions of Roumieu-Carleman type). In this space the set of universal functions is dense and $G_{\delta}$, although the space does not satisfy the Baire property. Moreover the two last new kinds of universal series cannot, in addition to the universal approximation, simultaneously satisfy the approximation property in the space where the universal functions should live. Based on this remark we return to an abstract theory. We give an abstract framework for this behavior from which we derive an approximation property concerning polynomials in the $C^{\infty}$ case or the $C^{\infty}$-Beurling case, which is analogous to a classical lemma of du Bois Reymond.

Finally we give a further extension of Fekete's Theorem in the $C^{\infty}$ class or in the $C^{\infty}$ class of Beurling ultradifferentiable functions. Now the Fekete property holds, not only for one center, but for a denumerable set of isolated points. The universal approximation is realized by the same sequence of indices according to a recent result on simultaneous approximation by Tsirivas [16].

\section{UNIVERSALITy IN A SPACE OF FORMAL POWER SERIES of Beurling TYPE}

Let us recall the abstract theory of universal series [12, 1]. Let $X$ be a metrizable topological vector space over the field $\mathbb{K}=\mathbb{R}$ or $\mathbb{C}$. Let $\rho$ be a translation invariant metric compatible with the vector space operations of $X$. Let $\left(x_{k}\right)_{k=0}^{+\infty}$ be a fixed sequence in $X$.

Definition 2.1. A sequence $a=\left(a_{0}, a_{1}, \ldots\right) \in \mathbb{K}^{\mathbb{N}}$ is said to belong to the class $\mathcal{U}$ if the sequence $\sum_{j=0}^{n} a_{j} x_{j}, n=0,1,2, \ldots$ is dense in $X$.

In other words, $\mathcal{U}$ is the set of unrestricted universal series. We are also interested in restricted universal series. We fix a subspace $A$ of $\mathbb{K}^{\mathbb{N}}$ and assume that it carries a complete metrizable vector space topology induced by a translation invariant metric $d$ such that the following three properties hold:

$\left(P_{1}\right)$ the coordinate projections $A \rightarrow \mathbb{K}, a \mapsto a_{m}$ are continuous for all $m \in \mathbb{N}$,

$\left(P_{2}\right)$ the set of polynomials $G=\left\{a=\left(a_{n}\right)_{n \geq 0} \in \mathbb{K}^{\mathbb{N}}:\left\{n ; a_{n} \neq 0\right\}\right.$ is finite $\}$ is contained in $A$,

$\left(P_{3}\right) G$ is dense in $A$.

As usual we denote by $\left(e_{n}\right)_{n \geq 0}$ the canonical basis of $\mathbb{K}^{\mathbb{N}}$.

Definition 2.2. A sequence $a \in A$ belongs to the class $\mathcal{U}_{A}$ if, for every $x \in X$, there exists a sequence $\left(\lambda_{n}\right)$ in $\mathbb{N}$ such that

$$
\sum_{j=0}^{\lambda_{n}} a_{j} x_{j} \rightarrow x \text { as } n \rightarrow+\infty \text { and } \sum_{j=0}^{\lambda_{n}} a_{j} e_{j} \rightarrow a \text { as } n \rightarrow+\infty .
$$

It is obvious that $\mathcal{U}_{A} \subset \mathcal{U} \cap A$. If we suppose that for every $a \in A, \sum_{j=0}^{n} a_{j} e_{j} \rightarrow a$ as $n \rightarrow+\infty$, then one has $\mathcal{U}_{A}=\mathcal{U} \cap A$. 
In the following, we need a further result in the abstract theory of universal series. We use the notations and definitions of section 1.3 of [1] with the simplification $L=$ $\{0\}$ and $E=A$. We assume that $\left(X_{k}\right)_{k \geq 1}$ is a sequence of metrizable topological vector spaces over $\mathbb{K}$ equipped with translation invariant metrics $\rho_{k}$. Let $\left(x_{k, n}\right)_{n=0}^{+\infty}$ be a fixed sequence in $X_{k}$.

Definition 2.3. Under the above assumptions, an element $a \in A$ belongs to the class $\mathcal{U}_{A}^{\mu}$, where $\mu$ is an increasing sequence of positive integers, if for every $k \geq 1$ and every $x \in X_{k}$, there exists a subsequence $\left(\lambda_{n}\right)$ of $\mu$ such that

$$
\rho_{k}\left(\sum_{j=0}^{\lambda_{n}} a_{j} x_{k, j}, x\right) \rightarrow 0 \text { as } n \rightarrow+\infty \text { and } d\left(\sum_{j=0}^{\lambda_{n}} a_{j} e_{j}, a\right) \rightarrow 0 \text { as } n \rightarrow+\infty .
$$

In the case $\mu=\mathbb{N}$, we use the notation $\mathcal{U}_{A}$.

Theorem 2.4 ([1]). Under the above assumptions the following are equivalent:

(1) $\mathcal{U}_{A} \neq \emptyset$

(2) For every $k \geq 1$, every $x \in X_{k}$ and every $\varepsilon>0$, there exist $n \in \mathbb{N}$ and $a_{0}, \ldots, a_{n} \in \mathbb{K}$ so that

$$
\rho_{k}\left(\sum_{j=0}^{n} a_{j} x_{k, j}, x\right)<\varepsilon \text { and } d\left(\sum_{j=0}^{n} a_{j} e_{j}, 0\right)<\varepsilon .
$$

(3) For any increasing sequence $\mu$ of positive integers, $\mathcal{U}_{A}^{\mu}$ is a dense $G_{\delta}$ subset of $A$.

(4) For any increasing sequence $\mu$ of positive integers, $\mathcal{U}_{A}^{\mu} \cup\{0\}$ contains a dense vector subspace of $A$.

In this section we apply this abstract theory of universal series to obtain a result of universality in the space of formal power series with control on the growth of coefficients. We use the following notation.

Notation 2.5. For any multi-index $J=\left(j_{1}, \ldots, j_{l}\right)$ of $\mathbb{N}^{l}$, we denote the length $j_{1}+\cdots+j_{l}$ by $|J|$ and we write $D^{J}=\partial^{|J|} / \partial x_{1}^{j_{1}} \ldots \partial x_{l}^{j_{l}}, x^{J}=x_{1}^{j_{1}} \ldots x_{l}^{j_{l}}$ and $J !=j_{1}$ ! $\ldots j_{l}$ !. Let $M=\left(M_{n}\right)_{n \geq 0}$ be an increasing sequence of real numbers with $M_{0}=1$. Denote by $\Lambda_{(M)}$ the set

$$
\Lambda_{(M)}=\left\{a=\left(a_{J}\right)_{J \in \mathbb{N}^{l}}, a_{J} \in \mathbb{K} ; \quad \forall C>0 \quad\|a\|_{C}^{(M)}=\sup _{J \in \mathbb{N}^{l}} \frac{\left|a_{J}\right|}{C^{|J|} M_{|J|}}<\infty\right\} .
$$

In the same spirit, denote by $\mathcal{F}_{(M)}$ the set of formal power series $f=\sum_{J \in \mathbb{N}^{l}} f_{J} x^{J}$ with $\left(f_{J}\right) \in \Lambda_{(M)}$. In particular the sequence $M$ can be considered as the defect of analyticity of the power series $f$.

Clearly $\Lambda_{(M)}$ (or $\left.\mathcal{F}_{(M)}\right)$ endowed with the topology given by the family of seminorms $\|.\|_{C}^{(M)}$ is a Fréchet space. For $a, b \in \Lambda_{(M)}$, the associated distance is defined by $d(a, b)=\sum_{i=0}^{+\infty} \frac{1}{2^{i}} \frac{\|a-b\|_{\sigma_{i}}^{(M)}}{1+\|a-b\|_{\sigma_{i}}^{(M)}}$, where $\left(\sigma_{i}\right)_{i \geq 0}$ is a strictly decreasing sequence of real numbers which converges to 0 . Moreover it is easy to check that properties $\left(P_{1}\right)$ and $\left(P_{2}\right)$ hold in $\Lambda_{(M)}$. The following lemma shows that $\left(P_{3}\right)$ is also true.

Lemma 2.6. The set of polynomials $G$ is dense in $\Lambda_{(M)}\left(\right.$ or $\left.\mathcal{F}_{(M)}\right)$. 
Proof. Let $a=\left(a_{J}\right)_{J \in \mathbb{N}^{l}}$ be a sequence in $\Lambda_{(M)}$ and let $\varepsilon>0$. There exists $m \in \mathbb{N}$ such that $\sum_{i=m+1}^{+\infty} 2^{-i}<\varepsilon / 2$. Let $0<\eta<1$ be a real number such that $2 \eta \sum_{i=0}^{m} 2^{-i}<\varepsilon$. Since $a$ belongs to $\Lambda_{(M)}$, there exists $C>0$ such that for every $J \in \mathbb{N}^{l}$, the following estimate holds:

$$
\frac{\left|a_{J}\right|}{\sigma_{m}^{|J|} M_{|J|}} \leq \frac{C}{2^{|J|}}
$$

Therefore there exists $j_{0} \in \mathbb{N}$ such that for every $J \in \mathbb{N}^{l}$, with $|J| \geq j_{0}$, we have $\frac{\left|a_{J}\right|}{\sigma_{m}^{J \mid} M_{|J|}}<\frac{\eta}{1-\eta}$. We define the polynomial $b=\left(b_{J}\right)_{J \in \mathbb{N}^{l}}$ by setting $b_{J}=a_{J}$ if $|J|<j_{0}$ and $b_{J}=0$ if $|J| \geq j_{0}$. We have by construction $\|a-b\|_{\sigma_{m}}^{(M)}<\frac{\eta}{1-\eta}$ and

$$
d(a, b)<\sum_{i=0}^{m} 2^{-i} \eta+\sum_{i=m+1}^{+\infty} 2^{-i}<\frac{\varepsilon}{2}+\frac{\varepsilon}{2}=\varepsilon .
$$

Notation 2.7. Fix an increasing sequence $\left(K_{k}\right)$ of compact subsets of $\mathbb{R}^{l}$ such that every compact set $K \subset \mathbb{R}^{l}$ is contained in some $K_{k}$. Then, for every $k \geq 1$, let $X_{k}$ be the space $C\left(K_{k}\right)$ endowed with the norm $\sup _{x \in K_{k}}|$.$| .$

We set $x_{k, J}=x^{J}$ and we will show that condition (2) of Theorem 2.4 is satisfied. First of all we need to introduce several definitions.

Definition 2.8. Let $M$ be an increasing sequence of real numbers with $M_{0}=1$. An infinitely differentiable function $f$ on $\mathbb{R}^{l}$ is called an ultradifferentiable function of class $M$ of Beurling type if on each compact set $K$ in $\mathbb{R}^{l}$ its derivatives satisfy the estimate

$$
\|f\|_{C, K}^{(M)}=\sup _{\substack{J \in \mathbb{N}^{l} \\ x \in K}} \frac{\left|D^{J} f(x)\right|}{|J| ! M_{|J|} C^{|J|}}<+\infty,
$$

where (11) holds for every $C>0$. We denote by $\mathcal{E}_{(M)}\left(\mathbb{R}^{l}\right)$ the set of ultradifferentiable functions $f$ of Beurling class $M$. It is clearly a Fréchet space (endowed with the topology given by the seminorms $\left.\|\cdot\|_{C, K}^{(M)}\right)$.

Moreover we will always impose the following conditions on the sequence $M$ :

$\left(H_{1}\right) \quad$ the sequence $M$ is logarithmically convex, i.e. $M_{n+1} / M_{n}$ increases;

$$
\sup _{p \geq 1, q \geq 1}\left(\frac{M_{p+q}}{M_{p} M_{q}}\right)^{\frac{1}{p+q}}<+\infty ;
$$

and there is a constant $C>0$ such that

$$
\sum_{n \geq p} \frac{M_{n}}{(n+1) M_{n+1}} \leq C \frac{M_{p}}{M_{p+1}}, p=1,2, \ldots
$$

Assumption $\left(H_{1}\right)$ implies stability under pointwise product. Condition $\left(H_{2}\right)$ guarantees stability under the action of ultradifferentiable operators 9. Hypothesis $\left(H_{3}\right)$ is known as the strong non-quasianalyticity. According to a result of Petzsche, condition $\left(H_{3}\right)$ is equivalent to the surjectivity of the Borel map: $\mathcal{E}_{(M)}\left(\mathbb{R}^{l}\right) \rightarrow \mathcal{F}_{(M)}$, $f \mapsto \sum_{J \in \mathbb{N}^{l}} \frac{D^{J} f(0)}{J !} x^{J}$ [14. In the sequel we shall use the following Weierstrass type result proved by Komatsu: under the above assumptions, the set of polynomials is dense in $\mathcal{E}_{(M)}\left(\mathbb{R}^{l}\right)(\underline{9}$, Theorem 7.3). 
Example 2.9. Let $\alpha$ be a real number, with $\alpha>0$. We set $M_{n}=(n !)^{\alpha}$. Then the sequence $M$ is strongly non-quasianalytic. This is the Gevrey $G^{1+\alpha}$ regularity, well-known in partial differential equations.

Lemma 2.10. Let $K \subset \mathbb{R}^{l}$ be a compact set. For every $\varepsilon>0$ and every $g \in C\left(\mathbb{R}^{l}\right)$ with $g(0)=0$ there exists a polynomial $p(x)=\sum_{J \in \mathbb{N}^{l}} p_{J} x^{J}$ with $p(0)=0$ such that

$$
\sup _{x \in K}|p(x)-g(x)|<\varepsilon \text { and } d\left(\left(p_{J}\right), 0\right)<\varepsilon,
$$

where $d$ is the distance in the Fréchet space $\Lambda_{(M)}$.

Proof. Let $\tilde{K}=[-C, C]^{l}$ be a compact set with $K \subset \tilde{K}$ and $C \geq 2$. First we apply the well-known Weierstrass approximation theorem to find a polynomial $q$ so that $\sup _{\tilde{K}}|q-g|<\varepsilon / 6$. Let us define the polynomial $\tilde{q}(x)=q(x)-q(0)$. By the triangle inequality we have $\sup _{\tilde{K}}|\tilde{q}-g|<\varepsilon / 3$. Since $\tilde{q}(0)=0$, we find $\delta \in(0, C]$ such that $|\tilde{q}(x)|<\varepsilon / 3$ for $x \in[-\delta, \delta]^{l}$. Let $\left.\Omega=\right]-C-1, C+1\left[{ }^{l} \backslash\left[-\frac{3 \delta}{4}, \frac{3 \delta}{4}\right]^{l} \subset \mathbb{R}^{l}\right.$ be an open set. There exists a function $\varphi \in \mathcal{E}_{(M)}\left(\mathbb{R}^{l}\right)$ with compact support in $\Omega$ such that $\varphi \equiv 1$ in a neighborhood of $\left.[-C, C]^{l} \backslash\right]-\delta, \delta\left[^{l}\right.$ and $|\varphi| \leq 1$ (9], Lemma 5.1). Let us consider the function $\tilde{q} \varphi \in \mathcal{E}_{(M)}\left(\mathbb{R}^{l}\right)$. There exists $m \in \mathbb{N}$ such that $\sum_{i=m+1}^{+\infty} 2^{-i}<\varepsilon / 2$. Let $0<\eta<1$ be a real number such that $6 \eta \sum_{i=0}^{m} 2^{-i}<\varepsilon$. We now apply the Weierstrass type theorem in $\mathcal{E}_{(M)}\left(\mathbb{R}^{l}\right) \underline{9}$ to find a polynomial $\tilde{p}$ such that

$$
\sup _{\substack{J \in \mathbb{N}^{l} \\ x \in \tilde{K}}} \frac{\left|D^{J}(\tilde{q} \varphi-\tilde{p})(x)\right|}{\sigma_{m}^{|J|}|J| ! M_{|J|}}<\frac{\eta}{1-\eta} .
$$

Using (2) with $J=0$ we get $\sup _{\tilde{K}}|\tilde{p}-\tilde{q} \varphi|<\varepsilon / 6$. We put $p(x)=\tilde{p}(x)-\tilde{p}(0)$ and $\tilde{K}=I_{1}(\delta) \cup I_{2}(\delta) \cup I_{3}(\delta, C)$ with $I_{1}(\delta)=\left[-\frac{\delta}{2}, \frac{\delta}{2}\right]^{l}, I_{2}(\delta)=[-\delta, \delta]^{l} \backslash\left(I_{1}(\delta)\right)^{\circ}$ and $\left.I_{3}(\delta, C)=[-C, C]^{l} \backslash\right]-\delta, \delta\left[{ }^{l}\right.$. By the triangle inequality we have $\sup _{\tilde{K}}|p-\tilde{q} \varphi|<$ $\varepsilon / 3$. Combining this inequality with the properties of the function $\varphi$ we derive the following estimates:

$$
\begin{aligned}
& \sup _{x \in I_{2}(\delta)}|\tilde{q}(x)-p(x)| \leq \sup _{x \in I_{2}(\delta)}|\tilde{q}(x) \varphi(x)-p(x)|+\sup _{x \in I_{2}(\delta)}|\tilde{q}(x) \varphi(x)-\tilde{q}(x)|<\frac{2 \varepsilon}{3}, \\
& \sup _{x \in I_{3}(\delta, C)}|\tilde{q}(x)-p(x)|=\sup _{x \in I_{3}(\delta, C)}|\tilde{q}(x) \varphi(x)-p(x)|<\frac{\varepsilon}{3} .
\end{aligned}
$$

$$
\sup _{x \in I_{1}(\delta)}|\tilde{q}(x)-p(x)| \leq \sup _{x \in I_{1}(\delta)}|\tilde{q}(x)|+\sup _{x \in I_{1}(\delta)}|p(x)|<\frac{2 \varepsilon}{3},
$$

From (3), (41) and (5) we get

$$
\sup _{x \in K}|p(x)-g(x)| \leq \sup _{x \in K}|p(x)-\tilde{q}(x)|+\sup _{x \in K}|\tilde{q}(x)-g(x)|<\frac{\varepsilon}{3}+\frac{2 \varepsilon}{3}=\varepsilon .
$$

Finally combining the equality $\left|D^{J}(q \varphi-p)(0)\right|=\left|p_{J}\right| J$ ! with inequality (2) we get $d\left(\left(p_{J}\right), 0\right)<\varepsilon$.

Combining Lemma 2.10 with Theorem 2.4 we deduce the following result.

Theorem 2.11. Let $l \geq 1$ be a fixed natural number and let $\gamma^{j}, j \geq 0$, be an arbitrary enumeration of the set of multi-indices $\gamma \in \mathbb{N}^{l}$, with $\gamma^{0}=(0, \ldots, 0)$. Let $\mu$ be an infinite subset of $\mathbb{N}$ and let $M=\left(M_{n}\right)_{n>0}$ be an increasing sequence of real numbers with $M_{0}=1$ satisfying the conditions $\left(H_{i}\right)(i=1,2,3)$. 
Then there exists a formal power series $f(x)=\sum_{j \in \mathbb{N}} a_{j} x^{\gamma^{j}} \in \mathcal{F}_{(M)}$ with $a_{0}=0$ which is universal in the following sense: for any continuous function $h: \mathbb{R}^{l} \rightarrow \mathbb{R}$ with $h(0)=0$ there exists a subsequence $\left(\lambda_{n}\right)$ of $\mu$ such that

$$
\sum_{j=1}^{\lambda_{n}} a_{j} x^{\gamma^{j}} \rightarrow h(x) \text {, as } n \rightarrow+\infty \text {, uniformly on each compact subset of } \mathbb{R}^{l} .
$$

The set $\mathcal{U}_{\Lambda_{(M)}}^{\mu}$ of such formal power series is $G_{\delta}$ and dense in the space of all formal power series in $\mathcal{F}_{(M)}$ without constant term and contains a dense vector subspace apart from 0.

Remarks 2.12. (1) Since $a=\lim _{n \rightarrow+\infty} \sum_{j=0}^{n} a_{j} e_{\gamma^{j}}$, one has $\mathcal{U}_{\Lambda_{(M)}}=\mathcal{U} \cap \Lambda_{(M)}$.

(2) Notice that the power series obtained obviously has radius of convergence 0 .

(3) By choosing in an appropriate way the enumeration $\left(\gamma^{j}\right)$ of the set of multi-indices and the infinite subset $\mu$ of $\mathbb{N}$, it is possible to assume that $\sum_{j=0}^{\lambda_{n}} a_{j} x^{\gamma^{j}}$ is of the form $\sum_{|J| \leq \lambda_{n}} a_{J} x^{J}$.

(4) Applying a translation, we can easily replace the assumption $h(0)=0$ by $h(0)=c \in \mathbb{R}$. In the same way, there are series that are universal for all continuous functions taking a fixed value $c$ at 0 and in this case $a_{0}=c$, of course.

(5) Finally it is possible to omit the initial condition on $h$ if we just consider the uniform convergence on each compact subset $K \subset \mathbb{R}^{l}$ with $0 \notin K$.

\section{UNIVERSALITY IN CLASSES OF $C^{\infty}$ FUNCTIONS}

First we give a version of Theorem 2.11 in $\mathcal{E}_{(M)}\left(\mathbb{R}^{l}\right)$. We refer to Definition 2.8 for the definition and properties of the space $\mathcal{E}_{(M)}\left(\mathbb{R}^{l}\right)$.

Theorem 3.1. Let $l \geq 1$ be a fixed natural number and let $\gamma^{j}, j \geq 0$, be an arbitrary enumeration of the set of multi-indices $\gamma \in \mathbb{N}^{l}$, with $\gamma^{0}=(0, \ldots, 0)$. Let $\mu$ be an infinite subset of $\mathbb{N}$ and let $M=\left(M_{n}\right)_{n \geq 0}$ be an increasing sequence of real numbers with $M_{0}=1$ satisfying conditions $\left(H_{i}\right)(i=1,2,3)$.

Then there exists a $C^{\infty}$ function $f \in \mathcal{E}_{(M)}\left(\mathbb{R}^{l}\right)$ with $f(0)=0$ which is universal in the following sense: for any continuous function $h: \mathbb{R}^{l} \rightarrow \mathbb{R}$ with $h(0)=0$ there exists a subsequence $\left(\lambda_{n}\right)$ of $\mu$ such that

$$
\sum_{j=0}^{\lambda_{n}} \frac{D^{\gamma^{j}} f(0)}{\gamma^{j} !} x^{\gamma^{j}} \rightarrow h(x) \text {, as } n \rightarrow+\infty \text {, uniformly on each compact subset of } \mathbb{R}^{l} \text {. }
$$

The set $\mathcal{U}^{\mu}\left(\mathcal{E}_{(M)}\right)$ of such functions is $G_{\delta}$ and dense in the space of all functions in $\mathcal{E}_{(M)}\left(\mathbb{R}^{l}\right)$ that vanish at 0 and contains a dense vector subspace apart from 0.

Proof. Let $\hat{f}=\sum_{J \in \mathbb{N}^{l}} f_{J} x^{J}$ be an element of $\mathcal{U}_{\Lambda_{(M)}}^{\mu}$. Since the Borel map $T_{0}$ : $\mathcal{E}_{(M)}\left(\mathbb{R}^{l}\right) \rightarrow \mathcal{F}_{(M)}$ given by $f \mapsto \sum_{J \in \mathbb{N}^{l}} \frac{D^{J} f(0)}{J !} x^{J}$ is surjective [14, there exists $f \in$ $\mathcal{E}_{(M)}\left(\mathbb{R}^{l}\right)$ satisfying $f_{J}=\frac{D^{J} f(0)}{J !}$ for every $J \in \mathbb{N}^{l}$. Therefore we have $\mathcal{U}^{\mu}\left(\mathcal{E}_{(M)}\right) \neq \emptyset$. Let $P$ be a polynomial with $P(0)=0$. Obviously $f+P$ belongs to $\mathcal{U}^{\mu}\left(\mathcal{E}_{(M)}\right)$. Combining this fact with the density of polynomials in $\mathcal{E}_{(M)}\left(\mathbb{R}^{l}\right)[9$, we deduce the following result: the set $\mathcal{U}^{\mu}\left(\mathcal{E}_{(M)}\right)$ is dense in the space of all functions in $\mathcal{E}_{(M)}\left(\mathbb{R}^{l}\right)$ that vanish at 0 . Since $\mathcal{E}_{(M)}\left(\mathbb{R}^{l}\right)$ is a Baire space, it suffices to apply Theorem 1 
of [7] to conclude that the class $\mathcal{U}^{\mu}\left(\mathcal{E}_{(M)}\right)$ is $G_{\delta}$. Finally observe that $\mathcal{E}_{(M)}\left(\mathbb{R}^{l}\right)$ is separable (9, Theorem 2.6). Hence to construct a dense vector subspace in $\mathcal{U}^{\mu}\left(\mathcal{E}_{(M)}\right)$ we proceed as in the proof of $(3) \Rightarrow(4)$ of Theorem 3 of [1] with the obvious modifications.

Remarks 3.2. (1) We claim that the elements $f$ of $\mathcal{U}^{\mu}\left(\mathcal{E}_{(M)}\right)$, in addition to the universal approximation, cannot simultaneously satisfy the following

$$
\sum_{j=0}^{\lambda_{p}} \frac{D^{\gamma^{j}} f(0)}{\gamma^{j} !} x^{\gamma^{j}} \rightarrow f, \text { as } p \rightarrow+\infty, \text { in } \mathcal{E}_{(M)}\left(\mathbb{R}^{l}\right) .
$$

Indeed we show that condition (2) in Theorem 3 of [1] is not satisfied. Let us recall this condition in our situation: for every $k \geq 1$, every $f \in X_{k}$ (that is, the space $C\left(K_{k}\right)$ endowed with the norm $\sup _{x \in K_{k}}|$.$| , where the$ sequence $\left(K_{k}\right)$ is defined in 2.7) and every $\varepsilon>0$, there exist $n \in \mathbb{N}$ and $a_{0}, \ldots, a_{n} \in \mathbb{R}$ so that

$$
\sup _{x \in K_{k}}\left|\sum_{j=0}^{n} a_{j} x^{\gamma^{j}}-f(x)\right|<\varepsilon \text { and } d_{\mathcal{E}}\left(\sum_{j=0}^{n} a_{j} x^{\gamma^{j}}, 0\right)<\varepsilon,
$$

where $d_{\mathcal{E}}$ is the distance in the Fréchet space $\mathcal{E}_{(M)}\left(\mathbb{R}^{l}\right)$. In our case, this condition means that the polynomial $\sum_{j=0}^{n} a_{j} x^{\gamma^{j}}$ realizes two different approximations on the same compact set. This is a contradiction.

(2) As in 2.12 we can obtain other universal elements by replacing the assumption $h(0)=0$ by $h(0)=c \in \mathbb{R}$ or by omitting the initial condition on $h$ if we just consider the uniform convergence on each compact subset $K \subset \mathbb{R}^{l}$ with $0 \notin K$.

Let us introduce another class of $C^{\infty}$ functions.

Definition 3.3. Let $M$ be an increasing sequence of real numbers with $M_{0}=1$. An infinitely differentiable function $f$ on $\mathbb{R}^{l}$ is called an ultradifferentiable function of class $M$ of Roumieu-Carleman type if on each compact set $K$ in $\mathbb{R}^{l}$ its derivatives satisfy the estimate

$$
\|f\|_{C, K}^{(M)}=\sup _{\substack{J \in \mathbb{N}^{l} \\ x \in K}} \frac{\left|D^{J} f(x)\right|}{|J| ! M_{|J|} C^{|J|}}<+\infty
$$

where (7) holds for some $C>0$. We denote by $\mathcal{E}_{\{M\}}\left(\mathbb{R}^{l}\right)$ the set of ultradifferentiable functions $f$ of Roumieu-Carleman type of class $M$.

The space $\mathcal{E}_{\{M\}}\left(\mathbb{R}^{l}\right)$ has a rather complicated locally convex structure. In particular it is non-metrizable and it is not a Baire space. However, the set of polynomials remains dense in $\mathcal{E}_{\{M\}}\left(\mathbb{R}^{l}\right)$ (9], Lemma 7.1 and Theorem 7.3). Observe that one has that

$$
\mathcal{E}_{(M)}\left(\mathbb{R}^{l}\right) \subset \mathcal{E}_{\{M\}}\left(\mathbb{R}^{l}\right) \subset C^{\infty}\left(\mathbb{R}^{l}\right),
$$

with dense continuous inclusions; note that each space contains the polynomials densely. Then Theorem 3.1 immediately implies that in $\mathcal{E}_{\{M\}}\left(\mathbb{R}^{l}\right)$ or $C^{\infty}\left(\mathbb{R}^{l}\right)$ one has dense sets and dense vector spaces of universal elements. The fact that the 
sets of universal elements are also $G_{\delta}$ is just due to the continuity of the derivative mappings. Indeed, let us define the sets

$$
\mathcal{O}_{\mu}(k, m, s, p)=\left\{f \in \mathcal{E}_{\{M\}}^{0}\left(\mathbb{R}^{l}\right) ; \sup _{x \in K_{k}}\left|\sum_{j=0}^{\mu_{p}} \frac{D^{\gamma^{j}} f(0)}{\gamma^{j} !} x^{\gamma^{j}}-f_{m}(x)\right|<\frac{1}{s}\right\},
$$

where $k, m, s=1,2, \ldots, p=0,1,2, \ldots,\left\{f_{m}\right\}$ is an enumeration of the polynomials of $n$ variables with rational coefficients without constant term and $\mathcal{E}_{\{M\}}^{0}\left(\mathbb{R}^{l}\right)$ is the set of functions $f \in \mathcal{E}_{\{M\}}\left(\mathbb{R}^{l}\right)$ with $f(0)=0$. Using the Weierstrass approximation theorem, it is easy to check that

$$
\bigcap_{m=1}^{\infty} \bigcap_{s=1}^{\infty} \bigcap_{k=1}^{\infty} \bigcup_{p=0}^{\infty} \mathcal{O}_{\mu}(k, m, s, p)=\mathcal{U}^{\mu}\left(\mathcal{E}_{\{M\}}\left(\mathbb{R}^{l}\right)\right)
$$

(see also 11] and 5, for proofs in the same spirit). Therefore the continuity of the linear map $\mathcal{E}_{\{M\}}\left(\mathbb{R}^{l}\right) \rightarrow C\left(K_{k}\right)$ given by $f \mapsto \sum_{j=0}^{\mu_{p}} \frac{D^{\gamma^{j}} f(0)}{\gamma^{j} !} x^{\gamma^{j}}$ ensures that the set $\mathcal{O}_{\mu}(k, m, s, p)$ is open in $\mathcal{E}_{\{M\}}\left(\mathbb{R}^{l}\right)$ for every $k, m, s$. The same proof works in $C^{\infty}\left(\mathbb{R}^{l}\right)$.

Finally, let us return to the abstract theory. Clearly the elements $f$ of $\mathcal{U}^{\mu}\left(C^{\infty}\left(\mathbb{R}^{l}\right)\right)$ (resp. $\left.\mathcal{U}^{\mu}\left(\mathcal{E}_{(M)}\left(\mathbb{R}^{l}\right)\right)\right)$ cannot, in addition to the universal approximation, simultaneously satisfy the approximation $\sum_{j=0}^{\lambda_{p}} \frac{D^{\gamma^{j}} f(0)}{\gamma^{j !}} x^{\gamma^{j}} \rightarrow f$, as $p \rightarrow+\infty$, in $C^{\infty}\left(\mathbb{R}^{l}\right)$ (resp. $\mathcal{E}_{(M)}\left(\mathbb{R}^{l}\right)$ ). There are other situations where $\mathcal{U}_{A} \neq \mathcal{U} \cap A$ (see [1], Example 6 ). Moreover the situation $\mathcal{U} \cap A \neq \emptyset$ is characterized by Theorem 30 of [1] when $A \subset \mathbb{K}^{\mathbb{N}}$. We can extend this result from the case of a space $A$ of sequences to a more general complete metrizable topological vector space. Then the existence of universal elements in $C^{\infty}\left(\mathbb{R}^{l}\right)$ or $\mathcal{E}_{(M)}\left(\mathbb{R}^{l}\right)$ becomes equivalent to the following polynomial approximation.

Lemma 3.4. For any $K \subset \mathbb{R}^{l}$ compact set, any continuous function $h: \mathbb{R}^{l} \rightarrow \mathbb{R}$ with $h(0)=0$, any function $w: \mathbb{R}^{l} \rightarrow \mathbb{R}$ of class $C^{\infty}\left(\mathbb{R}^{l}\right)$ (resp. $\mathcal{E}_{(M)}\left(\mathbb{R}^{l}\right)$ ) with $w(0)=0$ and for any $\varepsilon>0$, there exist $m, n \in \mathbb{N}, m \geq n$, and $a_{1}, \ldots, a_{m}$ in $\mathbb{R}$ such that

$$
\sup _{x \in K}\left|\sum_{j=1}^{n} a_{j} x^{\gamma^{j}}-h(x)\right|<\varepsilon \text { and } d_{\mathcal{E}}\left(\sum_{j=1}^{m} a_{j} x^{\gamma^{j}}, w\right)<\varepsilon,
$$

where $d_{\mathcal{E}}$ is the distance in the Fréchet space $C^{\infty}\left(\mathbb{R}^{l}\right)\left(\right.$ resp. $\left.\mathcal{E}_{(M)}\left(\mathbb{R}^{l}\right)\right)$.

The authors arrived at the statement of Lemma 3.4 using the abstract theory of universal series, extended to our situations, in combination with the existence of universal functions in $C^{\infty}\left(\mathbb{R}^{l}\right)$ and $\mathcal{E}_{(M)}\left(\mathbb{R}^{l}\right)$. It is natural to look for direct proofs also. In a personal communication, Kolountzakis has proved this result in the $C^{\infty}$ case without using the Borel theorem. Moreover the referee has given a direct proof of this lemma combining Lemma 2.10 with Borel's theorem. Finally observe that the statement of Lemma 3.4 is in the same spirit as the statement of a classical lemma of du Bois Reymond [2] ensuring the existence of a trigonometric polynomial $P=\sum_{j=-m}^{m} a_{j} e^{i j t}$ and an integer $n \leq m$ such that $\|P\|_{\infty}<\varepsilon$ and $\left|\sum_{j=-n}^{n} a_{j}\right|>x$, for $0<\varepsilon<x$. A famous result of Kolmogorov (see for instance [8] or [18, section 8.4] for a proof), which asserts that there exists a function $f \in L^{1}(\mathbb{T})$ such that, for each $t \in \mathbb{T}$, the sequence $\left(\sum_{|n| \leq N} \hat{f}(n) e^{i n t}\right)$ is unboundedly divergent, is also in 
the same spirit. Further the analogue of Lemma 3.4 for complex polynomials in $\mathbb{C}$ fails even if $w=0$ and $K=\{1\}$. If we have $\sup _{|z| \leq 2}\left|\sum_{j=0}^{m} a_{j} z^{j}\right| \leq 1, m \in \mathbb{N}$, then by the Cauchy estimates $\left|a_{j}\right| \leq \frac{1}{2^{j}}$, which implies $\left|\sum_{j=0}^{n} a_{j}\right| \leq 2$. Thus $\sum_{j=0}^{n} a_{j}$ cannot be close to 3 for any $n \in \mathbb{N}, n \leq m$.

\section{Further extensions of FeKete's theorem}

In Theorem 2.11 we can easily replace the assumption $h(0)=0$ by $h(0)=c \in \mathbb{R}$. We find series that are universal for all continuous functions taking a fixed value $c$ at 0 . Moreover one can generalize Theorem 2.11 by allowing arbitrary centers of expansion $a$. The set of such formal power series is also of the type $\mathcal{U}_{A}$; therefore the result of Tsirivas on simultaneous universal approximation can be applied [16]. We deduce the following result.

Proposition 4.1. Let $l \geq 1$ be a fixed natural number and let $\gamma^{j}, j \geq 0$, be an arbitrary enumeration of the set of multi-indices $\gamma \in \mathbb{N}^{l}$, with $\gamma^{0}=(0, \ldots, 0)$. Let $\mu$ be an infinite subset of $\mathbb{N}$ and let $M=\left(M_{n}\right)_{n \geq 0}$ be an increasing sequence of real numbers with $M_{0}=1$ satisfying conditions $\left(H_{k}\right)(k=1,2,3)$. Let $I$ be a finite or infinite denumerable set. We consider a family $a^{i} \in \mathbb{R}^{l}$ and $c^{i} \in \mathbb{R}, i \in I$. Then there exist formal power series $\sum_{j \in \mathbb{N}} a_{j}^{i}\left(x-a^{i}\right)^{\gamma^{j}} \in \mathcal{F}_{(M)}$ with $a_{0}^{i}=c^{i}$ having the following property: for every family of continuous functions $h^{i}: \mathbb{R}^{l} \rightarrow \mathbb{R}$ with $h^{i}\left(a^{i}\right)=0$ there exists a subsequence $\left(\lambda_{n}\right)$ of $\mu$ such that $\sum_{j=0}^{\lambda_{n}} a_{j}^{i}\left(x-a^{i}\right)^{\gamma^{j}} \rightarrow c^{i}+h^{i}(x)$ uniformly on each compact subset of $\mathbb{R}^{l}$ as $n \rightarrow+\infty$, for all $i \in I$.

We emphasize that the sequence $\left(\lambda_{n}\right)_{n=0}^{+\infty}$ is the same for all $i \in I$. The above result is generic and algebraically generic in naturally defined spaces (see [16]). We also note that the enumeration $\gamma^{j}, j=0,1, \ldots$, of the multi-indices may depend on $i \in I$. The same is valid for the statements that follow, but we will not insist on this point.

Now we consider a domain $\Omega \subset \mathbb{R}^{l}$ and a family $a^{i} \in \Omega, c^{i} \in \mathbb{R}, i \in I$, where the points $a^{i} \in \Omega, i \in I$ are distinct and the set $\left\{a^{i}: i \in I\right\}$ has no accumulation points in $\Omega$. Obviously the set $I$ is finite or infinite denumerable. The class $C^{\infty}(\Omega)$ and its topology do not need any explanation. The class $\mathcal{E}_{(M)}(\Omega)$ and its topology is analogous to $\mathcal{E}_{(M)}\left(\mathbb{R}^{l}\right)$ and its topology with the modification that the compact sets $K$ should satisfy $K \subset \Omega$. We will construct a function $f \in \mathcal{E}_{(M)}(\Omega)$ with $f\left(a^{i}\right)=c^{i}$ such that the Taylor developments of $f$ around $a^{i}$ satisfy Proposition 4.1 .

Since $a^{i}, i \in I$, does not have accumulation points in $\Omega$ we can find pairwise disjoint closed balls $\bar{B}\left(a^{i}, r^{i}\right) \subset \Omega, i \in I, r^{i}>0$ (for instance take $0<r^{i}<d^{i}$ with $d^{i}=\min \left(\operatorname{dist}\left(a^{i}, \Omega^{c}\right), \frac{1}{i}, \frac{1}{2} \min _{j \in I-\{i\}}\left|a^{i}-a^{j}\right|\right)$, where we assume $\left.I \subset\{1,2, \ldots\}\right)$. Then we can find $\varphi^{i} \in \mathcal{E}_{(M)}\left(\mathbb{R}^{l}\right)$ such that $\varphi^{i} \equiv 1$ on $\bar{B}\left(a^{i}, \frac{r^{i}}{2}\right)$ and supp $\varphi^{i} \subset B\left(a^{i}, r^{i}\right)$ 9]. Using Borel's theorem [14] we can find $f^{i} \in \mathcal{E}_{(M)}(\Omega)$ such that $\frac{D^{\gamma^{j}} f^{i}\left(a^{i}\right)}{\gamma^{j !}}=a_{j}^{i}$, $j \in \mathbb{N}, i \in I$. We set $f=\sum_{i \in I} \varphi^{i} f^{i}$. Since $\mathcal{E}_{(M)}(\Omega)$ is an algebra it follows that $\varphi^{i} f^{i} \in \mathcal{E}_{(M)}(\Omega)$. Since every compact set $K \subset \Omega$ meets only a finite number of the balls $\bar{B}\left(a^{i}, r^{i}\right), i \in I$, it follows easily that $f \in \mathcal{E}_{(M)}(\Omega)$. Thus we have proven the following.

Theorem 4.2. Let $\Omega \subset \mathbb{R}^{l}$ be a domain and let $a^{i} \in \Omega, i \in I$, be distinct such that the set $\left\{a^{i}: i \in I\right\}$ has no accumulation points in $\Omega$. Let $\gamma^{j}, j \geq 0$, be an 
arbitrary enumeration of the set of multi-indices $\gamma \in \mathbb{N}^{l}$, with $\gamma^{0}=(0, \ldots, 0)$. Let $\mu$ be an infinite subset of $\mathbb{N}$ and let $M=\left(M_{n}\right)_{n \geq 0}$ be an increasing sequence of real numbers with $M_{0}=1$ satisfying conditions $\left(H_{k}\right)(k=1,2,3)$. Let $c^{i} \in \mathbb{R}, i \in I$, be given. Then there exists $f \in \mathcal{E}_{(M)}(\Omega)$ with $f\left(a^{i}\right)=c^{i}, i \in I$, such that, for every selection $h^{i}: \mathbb{R}^{l} \rightarrow \mathbb{R}$ of continuous functions with $h^{i}\left(a^{i}\right)=0, i \in I$, there exists a subsequence $\left(\lambda_{n}\right)$ of $\mu$ such that $\sum_{j=0}^{\lambda_{n}} \frac{D^{\gamma^{j}} f\left(a^{i}\right)}{\gamma^{!}}\left(x-a^{i}\right)^{\gamma^{j}}, n \in \mathbb{N}$, converges to $c^{i}+h^{i}(x)$ uniformly on compact subsets of $\mathbb{R}^{l}$, as $n \rightarrow+\infty$, for all $i \in I$.

Using [1, part C], we conclude that the set of $f \in \mathcal{E}_{(M)}(\Omega)$ satisfying Theorem 4.2 is dense and $G_{\delta}$ in $\mathcal{E}_{(M)}(\Omega)$ and contains a dense vector subspace except 0 . The same holds in the space $C^{\infty}(\Omega)$. Finally what about the analogue of the RoumieuCarleman space on $\Omega$ ? Let us consider the class $\mathcal{E}_{\{M\}}(\Omega)$ of infinitely differentiable function $f$ on $\Omega$ of Roumieu-Carleman type. Since one has the dense continuous inclusions $\mathcal{E}_{(M)}(\Omega) \subset \mathcal{E}_{\{M\}}(\Omega) \subset C^{\infty}(\Omega)$, the set of $f$ 's in the Roumieu-Carleman space satisfying Theorem 4.2 is dense. The fact that this set of universal elements is also $G_{\delta}$ is just due to the continuity of the derivative mappings. We also obtain algebraic genericity.

Question. Let $f \in C^{\infty}(\mathbb{R})$ and $a \in \mathbb{R}$. We say that the point $a$ has Fekete's property for $f$ if for every continuous function $h: \mathbb{R} \rightarrow \mathbb{R}$ with $h(a)=0$ there exists a sequence $\left(\lambda_{n}\right)_{n=0}^{+\infty}$ in $\mathbb{N}$, so that $\sum_{j=0}^{\lambda_{n}} \frac{f^{(j)}(a)}{j !}(x-a)^{j} \rightarrow f(a)+h(x)$ uniformly on each compact subset of $\mathbb{R}$ as $n \rightarrow+\infty$.

The question that arises naturally is the following: Does there exist $f \in C^{\infty}(\mathbb{R})$ $\left(\right.$ or $\left.\mathcal{E}_{(M)}(\mathbb{R})\right)$ such that every $a \in \mathbb{R}$ has Fekete's property for $f$ ? Similar questions can be asked in $\mathbb{R}^{l}$ instead of $\mathbb{R}$.

\section{ACKNOWLEDGEMENTS}

The authors would like to thank K.-G. Grosse-Erdmann and the referee for helpful suggestions and J. Chaumat, M. Kolountzakis and V. Thilliez for useful communications.

\section{REFERENCES}

[1] F. Bayart, K.-G. Grosse-Erdmann, V. Nestoridis, C. Papadimitropoulos, Abstract theory of universal series and applications, Proc. London Math. Soc. 96 (2008) 417-463. MR 2396846 $(2009 \mathrm{j}: 30006)$

[2] P. du Bois Reymond, Über die Fourierschen Reihen, Nachr. Kön. Ges. Wiss. Göttingen 21 (1873) 571-582.

[3] E. Borel, Sur quelques points de la théorie des fonctions, Ann. Sci. École Norm. Sup. 12 (1895) 9-55. MR1508908

[4] C. Chui, M.N. Parnes, Approximation by overconvergence of power series, J. Math. Anal. Appl. 36 (1971) 683-696. MR0291472 (45:563)

[5] G. Costakis, M. Marias, V. Nestoridis, Universal Taylor series on open subsets of $\mathbb{R}^{n}$, Analysis 26 (2006) 401-409. MR2313217|(2008b:41026)

[6] K.-G. Grosse Erdmann, Holomorphe Monster und universelle Funktionen, Mitt. Math. Sem. Giersen 176 (1987) 1-84. MR877464 (88i:30060)

[7] K.-G. Grosse Erdmann, Universal families and hypercyclic operators, Bull. Amer. Math. Soc. (N.S.) 36 (1999) no. 3, 345-381. MR.1685272 (2000c:47001)

[8] J.P. Kahane, Baire's category theorem and trigonometric series, J. Anal. Math. 80 (2000) 143-182. MR1771526 (2001f:42012)

[9] H. Komatsu, Ultradistributions, I. Structure theorems and a characterization, J. Fac. Sci. Univ. Tokyo, Sect. 1A 20 (1973) 25-105. MR0320743(47:9277) 
[10] W. Luh, Approximation analytischer Funktionen durch uberkonvergente Potenzreihen und deren Matrix-Transformierten, Mitt. Math. Sem. Giessen 88 (1970) 1-56. MR0280692 (43:6411)

[11] V. Nestoridis, Universal Taylor series, Ann. Inst. Fourier (Grenoble) 46 (1996) no. 5, 12931306. MR1427126 (97k:30001)

[12] V. Nestoridis, C. Papadimitropoulos, Abstract theory of universal series and an application to Dirichlet series, C. R. Math. Acad. Sci. Paris 341 (2005) no. 9, 539-543. MR2181390 (2006h:11108)

[13] G. Pál, Zwei kleine Bemerkungen, Tokohu Math. J. 6 (1914/15) 42-43.

[14] H.-J. Petzsche, On E. Borel's theorem, Math. Ann. 282 (1988) 299-313. MR.963018 (89m:46076)

[15] A.I. Seleznev, On universal power series, Math. Sbornik N.S. 28 (1951) 453-460. MR0041928 $(13: 23 \mathrm{e})$

[16] N. Tsirivas, Simultaneous approximation by universal series, Math. Nachr., to appear (2010).

[17] H. Whitney, Analytic extensions of differentiable functions defined in closed sets., Trans. Amer. Math. Soc. 36 (1934) no. 1, 63-89. MR1501735

[18] A. Zygmund, Trigonometric Series, Cambridge University Press (1979).

Laboratoire de Mathématiques, UMR 8524, Cité Scientifique, 59650 Villeneuve D'ASCQ, France

Current address: École Centrale de Lille, Cité Scientifique, 59650 Villeneuve d'Ascq, France

E-mail address: Augustin.Mouze@math.univ-lillel.fr

Department of Mathematics, Panepistimiopilis, University of Athens, Athens, 15784, GREECE

E-mail address: vnestor@math.uoa.gr 